Get Your Coat: Examining the Development of Independent Dressing

Skills in Young Children with Visual Impairment, Down syndrome and Typically Developing Children

Submitted to the International Journal for Inclusive Education

Hayton, $\mathbf{J}^{1,2}$., Wall, $\mathrm{K}^{1}$., Dimitriou, $\mathrm{D}^{1,2}$.

${ }^{1}$ Department of Psychology and Human Development, UCL-Institute of Education, London, $U K$.

${ }^{2}$ Lifespan Learning and Sleep Laboratory, Department of Psychology and Human Development, UCL-Institute of Education, London, UK.

Correspondence details: Dr Jessica Hayton. Lifespan Learning and Sleep Lab, Department of Psychology and Human Development, UCL, Institute of Education, 25 Woburn Square, London, WC1H 0AA. Email: Jessica.hayton.14@ucl.ac.uk

Word count: 6279 including tables, figures and references. 


\title{
Get Your Coat: Examining the Development of Independent Dressing Skills in Young Children with Visual Impairment, Down syndrome and Typically Developing Children
}

\author{
Dressing is a fundamental independent living skill (ILS). Vision is an integrative \\ sense which affords learning via sighted observation. Visual impairment (VI) \\ denies/restricts access to learning via sight. As a result, children with VI and \\ conditions where VI may be a component (e.g. Down syndrome (DS)) require \\ structured, systematic support to develop ILS. The current study used a repeated- \\ measures-intervention approach to examine the development of the ILS of \\ dressing (ILSD) with novel intervention materials in young children. Participants \\ ( $N=27$ : age range 5;05-10;02 years) were drawn from three groups: VI ( $n=9$; age \\ range 5;05-10;02 years); DS ( $n=9$; age range 5;05-10;00 years) and Typically \\ Developing (TD) ( $n=9$; age range 6;05-8;00 years). The effectiveness of the \\ intervention materials was measured over a period of 10 weeks. Clear and \\ systematic verbal instruction combined with motor activities were useful in \\ supporting ILSD. The assessment of the longer term effectiveness was carried out \\ at 1 and 3 months post-intervention. This paper reported that the novel \\ intervention materials supported the development of ILSD in all three groups. \\ The findings could form a model for future work regarding the development of \\ ILS in other skill areas and generalised to other developmental disorders.
}

Keywords: habilitation; inclusion; visual impairment; independence; task analysis

\section{Introduction}

The current study examined the development of the independent living skill of dressing (ILSD) in two clinical groups, namely young children with Visual Impairment (VI), and young children with Down Syndrome (DS) in comparison to Typically Developing (TD) children.

Every day an individual has a need to get dressed. Hence, it might be assumed that as dressing is an integral part of everyday life, regardless of needs, previous research would have been undertaken in terms of finding the most effective ways of developing 
ways of dressing in developing children with a range of needs. Whether the act of dressing is carried out independently or not, society dictates that an individual should be dressed, despite the fact that we were all born naked. If individuals do not or are unable to dress themselves, somebody else, (i.e. a parent/caregiver) usually takes responsibility for dressing their child. This means that we either dress ourselves or are dressed by others.

The concept of dressing represents a developmental process in which parents and carers are the first dressers. They make the decisions about suitable clothing types to dress their child. The developmental process of dressing subsequently occurs through a child's social interactions with parents/caregivers (because there is very little formal, explicit, school based type teaching), and also taking opportunities to participate in the dressing routine. This paper argues that (in a typical context) the ILSD, viewed from a socialconstructivist perspective, is acquired through sighted observation and incidental learning. The process of acquired learning expertise is done experientially through extensive practising over a prolonged period of time.

The developing child gradually progresses by the parents' instruction and active participation in the dressing routine (see Kellegrew, 2000). The progressive development of ILSD occurs to the point of which, in optimal conditions, the child is increasingly able to dress himself/herself; transitioning from dependence to independence (Hayton, 2012). This incremental development leading to independent dressing skills is usually attained by the time the child enters a formal schooling environment.

One of the most important domains used in the typical development of ILSD is vision. Through a preserved visual modality, individuals are able to have an immediate and synchronised representation of their environment. Vision is also fundamental for perception and subsequent action such as moving toward and attending to objects as an environmental response (Sugden and Wade, 2013). Sighted children are able to develop 
ILS through indirect observations and mimicking, thus learning incidentally as a byproduct of an activity (Marsick and Watkins, 2001). For a child with vision, the observation of the actions of others appears to underpin the demonstration of modelling and imitative behaviours. These behaviours then support the development of motor skills. However, these modelling and imitative behaviours are often delayed or impaired in children where VI is diagnosed or is a component (Lewis, 1987), such as DS. This is because reduced visual functioning restricts or denies access to visual observation as a tool for learning. In addition to children clinically diagnosed with VI, children with DS were selected as another clinical group. This was due to the comorbidity of VI in a high percentage of the DS population (Creavin and Brown, 2009).

For those with identified needs such as VI and DS, the developmental trajectory of the child is: delayed by approximately 3 months (in the case of VI (Sugden and Wade, 2013; Warren, 1984)) or atypical (in the case of DS where cognitive delay is also a component) (Van Herwegen, Rundblad, Davelaar, and Annaz, 2011). Due to a knock out effect from physical, sensory or coordination delays or atypicalities, the acquisition of independent dressing skills may also be delayed. This is arguably linked to the development of motor skill and coordination required to independently execute the dressing task. Thus one would expect to see this domain carefully examined in the literature, however, this is not the case. In fact, there has been no systematic examination of the strategies and development to support independent dressing since the early 1950s. Several anecdotally acknowledged dressing strategies have been alluded to but not explicitly mentioned in relevant literatures (see: Fairnham, Johnston, Kain, Kain, McCauley and Steele, 2002; Bishop, 2004). This acknowledgement is done in relation to a barrier to developing adaptive behaviour, of which dressing is a sort (Sugden and Wade, 2013; Herbert, 2003). There is a suggestion therefore that an issue surrounding the 
development of dressing skills in the clinical population is apparent. It is argued here that this is perhaps most pertinent in the VI population and individuals with DS where visual problems are part of their clinical profile. Hence the focus of the current study was to examine if:

- Children with VI have reduced abilities or are unable to learn incidentally through sighted observation (Herbert, 2003)

- Children with VI have reduced abilities or are unable to use the visual modality to refine and control hand-eye and body coordination (Sugden and Wade, 2013),

- Children with VI are less likely to move around and explore their environment, subsequently restricting incidental haptic learning experiences (Bowman, Bowman, and Dutton, 2001)

Taking together the reasons stated above, the rationale for the current paper is to construct a systematic intervention protocol to support the ILSD. The development of the systematic intervention procedure was based on habilitation techniques. In terms of methodological approaches, habilitation techniques are used to aid the development of independence in individuals who have an impairment from birth (Miller, Wall, and Garner, 2011). The concept of habilitation is not a new idea and was previously discussed in the 1970s, however the term 'inclusion' became more popular within the literature. The conceptualisation of habilitation receded until the early 2000s when the term reemerged largely due to the publication of the Quality Standards (Miller, Wall, and Garner, 2011). Despite the reconceptualization of habilitation, there remains to be an appropriate, coherent, unifiable theory underpinning habilitation. Currently, the concept of habilitation has sought to begin to unify the previously distinct three components: orientation, mobility and ILS. 
By using habilitation techniques as a basis, the development of the ILSD protocol enabled the dressing process to be more carefully understood and executed within the schooling environment, and, incidentally, with parents. The schooling environment was chosen as attaining independence is a curriculum entitlement, and also, affords the opportunity for children to work in groups, which could encourage peer learning through social interaction.

Habilitation is now recognised within the English Special Educational Needs and Disabilities Code of Practice: 0-25 years (Department for Education and Department of Health, 2015), hence the intervention procedure in the current study was designed to incorporate play based learning. It is argued here that this could have an impact on the effectiveness of delivering a play-based intervention programme. Play based learning is argued to be effective in terms of appealing to incidental learning, and also is useful in supporting physical, social and emotional development of children. It is acknowledged, however, that play is not commonly incorporated within a traditional schooling environment (Moyles, 2015). Opportunities for play-based learning are usually adultdirected and occur at specific times through the school day (Moyles, 2015). This paper supports the notion that the multi-dimensional aspect of play facilitates movement, which initiates exploration, which in turn, facilitates learning. This supports the experiential aspect to the development of ILSD.

The overarching research question outlined for this study is: 'To what extent can novel intervention materials support the development of gross and fine motor skills suitable for independent dressing in children with VI in a range of needs and contexts?' In order to begin to answer this question, the current study involved a re-assessment of previous research and practice in the light of current and contemporary understandings of sensory and motor development in children. The research has also taken into account the 
historical background that has led to the changes of our understanding of sensory and motor development in children. This also involved developing novel interventions for habilitation programmes, rather than adapting previous interventions that are largely based on rehabilitation adult-based techniques (Miller, Wall, and Garner, 2011; Fairnham, Johnston, Kain, Kain, McCauley and Steele, 2002; Klein, 1983).

The current study therefore developed a systematic protocol based on task analysis which was used to support the ILSD. The task analysis approach splits a large task (e.g. fastening a button) into smaller, more nuanced yet observable stages (e.g. pinching a button between the index finger and the thumb). This was done to better understand the nuanced development of dressing skills over the intervention period, as each fastening task (e.g. fastening a zip, button, popper and shoe laces) was split into smaller, yet observable, sequences suitable for task completion. This sequential approach using clear and systematic instruction (see: Klein, 1983; Barrett and Bridson, 1983) toward the development of the ILSD could be indicative of skill development and acquisition in children with children with VI, DS (with VI) and TD children. One reason for the effectiveness of this approach may be because a child can become more motivated toward task completion when successfully achieving smaller tasks, rather than becoming de-motivated by being unable to complete the larger task.

Thus, the purpose of this study was to develop and test novel intervention materials to support ILSD development in children with VI and DS, and offer a contribution to the field of habilitation in terms of a systematic approach toward the development of ILSD. The current study is the first to empirically assess the immediate and longer-term impact of a novel intervention targeted at increasing ILSD in young children with VI and DS. 


\section{Methods}

\subsection{General Methods}

The participant sample was drawn from two clinical populations, namely VI and DS and one control TD group. Due to low prevalence rate of VI in the UK the recruitment was based on participant availability. The inclusion criteria were English as a first language due to the nature of the study, and current clinical diagnoses (for VI and DS groups). A background medical questionnaire was completed by parents/carers/teachers within the specialist schools/organisations contacted, after consent for participation was obtained.

The study was a mixed methods, repeated measures design. Participants were initially assessed at pre-intervention level. They were then assessed at weekly intervals over the 10-week intervention period. The impact of the 10-week assessment was measured after the intervention ceased. Follow up assessments were made 1 month and 3 months post-intervention to assess the long-term impact of the intervention.

The study worked in accordance with the BPS Code of Practice. Ethical approval was granted from the UCL-Institute of Education Ethics Committee. Child participants were informed as to the nature of the study, and were offered to participate before every contact session. Adult and child participants were informed of their right to withdraw from the project at any point during the research, and also that the collected data would be confidential and anonymised.

Since the current study examined children from different clinical populations, heterogeneity was assumed and as this has been reported previously. For instance, Van Herwegen et al. (2011) discussed high level of variance within clinical populations but also in the TD sample. Thus from the developmental approach heterogeneity is not problematic but rather a product of developmental changes. Hence, potential explanations of heterogeneity should be noted by, for example, incorporating individual participant 
characteristics within the research design. In the current study, full medical background profiles (including diagnosis and chronological age) were obtained via a variety of questionnaires. The questionnaires used are detailed in the apparatus section (section 2.3) of this study.

One further issue when working with VI populations was the availability and suitability of standardised measures. Previous studies exploring VI had a tendency to use measures designed for a TD population, making little or no modification to the resource (Warren, 1994). The current research used measures that were considered appropriate for the clinically defined groups and the TD group (see section 2.3).

A methodological issue was also raised in relation to the development of an intervention. This issue was "ceiling/floor effects". Ceiling effects typically occur when a task is considered to be too easy for completion and it is not possible to record sufficient differentiation of task performance in the samples. Floor effects may also occur within the intervention, as the task may be too difficult for participants to complete. In the current study, ceiling effects naturally occurred as a signifier of successful fastening abilities. Successful fastening/unfastening leaves no room for further progression within the scoring of the task. This is because the task ends when fastening/unfastening is complete. Ceiling effects, therefore, were the target scores for the recruited participants.

\subsection{Participants}

A total of 27 child participants were recruited for the current study. Eighteen participants were recruited from two clinically defined populations, namely VI and DS. The remaining 9 participants were TD control children. All participants fulfilled the inclusion criteria and were involved in the study as they all demonstrated a need to develop ILSD as they were unable to fasten zips, buttons, poppers and laces. 
Nine TD children (6 male; age range $=5 ; 06-7 ; 09$ years; $M=6 ; 09$ ) attended mainstream school located in Cumbria, England. At the time of testing, all TD participants were undiagnosed with any learning disabilities. Nine children with VI (6 male; age range 6;06-10;02 years; $M=8 ; 03$ ) were recruited from a specialist school in London, England. Eight children with VI had a clinical diagnosis of VI, one child was clinically undiagnosed but was reported to have partial sightedness. Diagnoses largely varied between participants with VI. Nine children with DS (4 male; age range: 5;0410;00 years; $M=7 ; 05$ years) were recruited from a voluntary organisation in London, England. Due to the auditory impairment associated with DS, the participants also used Makaton/British Sign Language to support their communication needs.

\subsection{Apparatus}

Background measures were used in order to minimise the effects of heterogeneity within each sample. The measures were also considered useful in informing the results obtained from the observation of progression over the intervention period. Background measures included a Medical History Questionnaire and a Socio-economic Questionnaire. Standardised tests of cognitive functioning suitable for all three recruited groups were also conducted. These were the Digit Span (taken from the WISC-IV battery), and Semantic and Phonemic Verbal Fluency (see: Strauss, Sherman, and Spreen, 2006).

Three novel intervention materials were also designed and created to support ILSD development. These were: the Interactive Puzzle Game (IPG); an interactive rhyming story (entitled "Just Joey"); and, a Standardised Outdoor Coat. The creation of these resources was due to a commercial unavailability for suitable resources. One commercially available resource was used to support the novel materials ("Learn to Dress with Alex") which for the purpose of the intervention was called "Joey". An observation 
schedule was created in order to assess participant progression over the intervention period. Table 1 shows the name, type and purpose of each resource.

[Table 1 near here]

\subsection{Procedure}

All recruited participants partook in a familiarisation period before the intervention commenced. This was considered important to support the children in engaging with the intervention and the researcher. Next, a screening procedure was carried out to ensure that the recruited participants would benefit from the intervention. The screening procedure confirmed that the recruited sample needed support in fastening zips, buttons, poppers and shoelaces. After confirmation of suitability for participation, the recruited samples participated in two 15-minute sessions per week over the 10 -week intervention period. Over the 10 weeks, the tasks increased in difficulty aiding the development of ILSD and also provided the opportunity for transference of ability from the abstract IPG to their actual outdoor coats. During the 10-week intervention procedure, assessments of participants' skills were made at weekly intervals using the previously piloted observation schedules (Hayton, 2016). After the 10-week intervention period, the VI group and the TD group were reassessed 1 month and 3 months post-intervention in order to examine the longer-term impact of the intervention on ILSD ability. The reassessment was not possible in the DS group due their absence from the group sessions. Many of the children were unable to attend the sessions due to either illness or holiday. Attendance to the sessions was voluntary as they were run outside of compulsory schooling hours (over the weekend). This had a direct impact on the set timings of the intervention, so post-testing for the DS group was not possible. 


\section{Results}

\subsection{Absence}

Participant absences were recorded over the intervention period as it became apparent that it may have an effect on the scores. Over the 10-week period, 14 cases of absence were reported in the VI group, and 19 absences were reported in the DS group. There were no reported absences in the TD group. Possible reasons for this are explored in the discussion section.

\subsection{Results of the Main Study}

Due to medical conditions, absence rates were very high in the clinically defined groups hence partial analyses of the data were used. The partial analyses examined weekly performance during the weeks when most participants were present, namely, preintervention (baseline) and weeks 4, 7 and 10. The differences between scores over these weeks were then transformed into a percentage against the ceiling score for independence. This means that the scores reported here are indicative of the percentage change (towards independence) based on the weekly scores where most participants were present. The conversion of the scores was also done to directly demonstrate the transition between dependence on adult support (represented by 100\%) and total independence (represented by $0 \%$ ). As some children reached their personal ceiling effects, which was demonstrated by zero percentage change between weeks as their observation scores remained constant, they were scored as $0 \% *$.

----Figure 1. Approx. here----

Examination of the intervention effects on zip fastening ability was done using ANOVA. The ANOVA revealed a noteworthy effect of the intervention on zip fastening ability; 
Wilks Lambda $=.117, F(3,13)=37.57, p=<.001$. In addition there was another noteworthy result between the zip improvement score and group; Wilks Lambda $=.116$, $F(6,26)=8.37, p=<.001$. This demonstrated that all three separate groups appeared to benefit from the intervention.

Paired samples t-tests were run to examine the effect of the percentage change within groups. Within each group, noteworthy findings were reported between baseline and week 1 scores $(\mathrm{VI} ; t(6)=3.31, p=.016$ : DS; $t(6)=4.01, p=.007: \mathrm{TD} ; t(8)=9.69$, $p=<.001)$. Noteworthy findings were only reported in the DS and the TD group between week 1 and week $4(\mathrm{DS} ; t(4)=6.57, p=.001$ : TD; $t(8)=9.69, p<.001$. Finally, a noteworthy finding was reported between week 4 and week 7 scores in the VI group $(t(4)=$ $2.92, p=.027)$.

-----Figure 2. Approx here-----

Examination of the intervention effects on button fastening ability was done using ANOVA. The ANOVA revealed a noteworthy effect of the intervention on button fastening ability; Wilks Lambda $=.016, F(3,14)=281.93, p=<.001$. In addition there was another interesting result between the button improvement score and group; Wilks Lambda $=.020, F(6,28)=28.71, p=<.001$. This demonstrated that all three separate groups appeared to benefit from the intervention.

Paired samples t-tests were run to examine the effect of the percentage change within groups. Within each clinical group (VI and DS), noteworthy results were reported between baseline and week 1 scores $(\mathrm{VI} ; t(6)=4.05, p=.007$ : DS; $t(5)=7.26, p=.001)$; week 1 and week $4(\mathrm{VI} ; t(5)=2.93, p=.001: \mathrm{DS} ; t(4)=6.57, p=.001: \mathrm{TD} ; t(8)=9.69$, $p<.001$. There were no reported differences in the VI or DS groups between week 4 and 
week 7. A paired samples t-test could not be carried out within the TD sample due to participants' independent fastening ability at baseline level.

------Figure 3. Approx here-----

Examination of the intervention effects on popper fastening ability was done using ANOVA. The ANOVA revealed a noteworthy effect of the intervention on popper fastening ability; Wilks Lambda $=.003, F(3,14)=1587.69, p=<.001$.

Paired samples t-tests were run to examine the effect of the percentage change within groups. Within the VI group, an interesting difference was reported between baseline and week 1 scores $(\mathrm{VI} ; t(6)=8.26, p=<.001)$. Noteworthy findings were found in the VI and DS groups between week 1 and week $4(\mathrm{VI} ; t(5)=3.81, p=.013$ : DS; $t(5)=$ 28.53, $p=<.001)$. An effect was also reported in the VI group between week 4 and week 7 scores $(\mathrm{VI} ; t(6)=2.64, p=.038)$ A paired samples t-test could not be carried out within the TD sample as there was no variance in performance.

-Figure 4. Approx here

Examination of the intervention effects on lace fastening ability was done using ANOVA. The ANOVA revealed a noteworthy effect of the intervention on lace fastening ability; Wilks Lambda $=.088, F(3,14)=48.10, p=<.001$. In addition there was another interesting result between the lace improvement score and group; Wilks Lambda $=.124$, $F(6,28)=8.60, p=<.001$. This demonstrated that all three separate groups benefited from the intervention. 
Paired samples t-tests were run to examine the effect of the percentage change within groups. Within each group, differences were reported between baseline and week 1 scores $(\mathrm{VI} ; t(6)=4.58, p=.004$ : DS; $t(6)=3.37, p=.015$ : TD; $t(8)=6.14, p=<.001)$; week 1 and week 4 scores $(\mathrm{VI} ; t(5)=2.93, p=.033$ : DS; $t(5)=4.63, p=.006: \mathrm{TD} ; t(8)=190.25$, $p<.001$. A noteworthy difference was only reported in the DS group between week 4 and week 7 scores $(\mathrm{DS} ; t(4)=3.50, p=.025)$.

Assessment of longer-term impact was carried out 1 month and 3 months postintervention in the VI and the TD groups. The following figures display the mean group scores in comparison to the week 10 observation. All four figures show that the independent fastening abilities remained to be at ceiling for the TD group. The VI group showed some different findings dependent on condition and length of time postintervention.

-Figure 5 approx. here-

Figure 5 shows that within the VI group mean, there was a slight increase in zip-fastening dependence 1 month post intervention. 3 months after intervention, the VI group increased toward independence without formal intervention. This is represented by the upward gradient toward $0 \%$.

--Figure 6. approx. here

Figure 6 shows that the VI group remained to be at ceiling for button fastening 1 month post-intervention, however demonstrated an increase in dependence 3 months postintervention. 
-Figure 7. Approx here

Figure 7 shows that the VI group continued to increase toward independent popper fastening despite the lack of exposure to the intervention. This is the only example of consistent participant improvement post-intervention.

Figure 8. Approx here

Figure 8 shows that 1 month post-intervention the VI group reduced in independence scores for lace fastening. However, 3 months post-intervention the group mean showed an increase in independence scores.

The data revealed that the intervention had a noticeable effect on the development of ILSD for all fastening conditions. This was most apparent in the differences between baseline and week 1 scores across all three groups. Over the intervention period, the values of the paired samples t-test decreased. This demonstrated a movement toward ceiling effects as the variance was reduced.

\section{Discussion}

The current study demonstrated that a 10-week intervention showed significant support in the development in ILSD in all three investigated groups (VI, DS and TD). However, the TD children acquired independence skills more rapidly than the clinically defined groups. This was expected given that the visual system was intact and with the assumption that incidental learning through sight would occur. Although the TD group acquired ILSD faster, the clinical groups displayed the largest improvement in independent fastening 
abilities over the 10-week period (Hayton, 2015). This is because the baseline for the clinical groups was lower than that of the TD group (with the exception of lace fastening).

The noteworthy findings supports the suitability of the use of novel intervention materials in the development of gross and fine motor skills suitable for ILSD in children with VI in a range of needs and contexts. The t-test results demonstrating the difference between week 4 and week 7 zip scores yielded interesting results. Within the VI paired samples t-test, a difference was found at this stage for the zip fastening condition. The noteworthy result indicated that the intervention appeared to increase independence in the VI group over this period of time thus suggesting the suitability of a 10 week intervention period for children with VI.

A suggested reason for the noteworthy result for VI participants at this stage of the intervention (week 4 - week 7, rather than between week 1 and week 4 scores) may have been due to the complexity of the zip fastening task. This is because in order to successfully fasten a zip, one must insert the tail into the slider. This aspect of the task demands fine motor control, co-ordination of the hands and the ability to pinch the fingers together. This is arguably the most complex stage of zip fastening, and so the noteworthy result that occurred between week 4 and week 7 may have been representative of the VI participants beginning to overcome these more complex stages of zip fastening. This difference was not apparent in the DS nor TD groups for the zip condition.

Arguably, the lack of difference between these scores in the DS group may have been due to DS participants failing to reach the ceiling scores for the condition. The desired outcome of the intervention was for participants to reach the ceiling scores within the intervention period. This was because the reaching of ceiling scores represented the ability to independently fasten/unfasten each fastening condition, which could have been suggestive of the effectiveness of the intervention procedure. Although the DS group did 
not reach the ceiling effects for the zip condition, it is posited that a 10-week intervention may also be reasonable for this group. This is because of the initial similar performance between the VI and DS groups and the high absence rates of the DS group.

In the current study, ceiling effects reflected a participant's independent skill ability in each fastening condition. This was considered important as the nature of the intervention task was to support each participant in reaching ceiling effects for each fastening condition. A ceiling score represented an individual's ability to independently fasten/unfasten a fastening condition. Ceiling effects over a number of weeks arguably demonstrated that the skill lasted for a longer period of time that may have been suggestive of the effectiveness of the intervention tasks.

An interesting finding was also noted between the week 4 and week 7 t-test scores in the popper condition within the VI group. This was due to an apparent increase in independent popper fastening skills over this time. The result could be further characterised by participants reaching the ceiling effect brought about by the fastening task. During week 4, three participants with VI had reached ceiling effects for the popper condition compared to five participants with VI reaching ceiling by week 7 . This arguably demonstrated that the 10-week intervention period was suitable for developing independent popper fastening abilities within the recruited VI group.

The exploration of the results showed that the novel intervention materials did appear to increase ILSD in the VI group who were characterised as having different diagnostic needs and personal contexts (as indicated by the background measures). The novel intervention materials also appeared to support the development of ILSD in DS and TD groups who also had different needs and contexts. The results suggested that the intervention materials and the length of time prescribed to the development of fastening ability were arguably effective in the development of ILSD in the specific recruited 
participant groups (VI, DS and TD). Based on the assessment of longer-term impact of the intervention, it could be assumed that the intervention had a lasting impact on motor skill acquisition suitable for ILSD in VI and TD groups.

\subsection{Limitations of the current study}

Absence was considered an important factor in the development of ILSD. Absence is arguably a characteristic of populations with profound and complex disabilities such as VI and DS. A further contributing factor to the higher level of absence in the DS group could have been the environment of which the intervention was carried out. As participants with DS were recruited from a voluntary organisation, attendance was not compulsory. Within the VI group, participant absence was a direct result of illness. It is important to note that all recoded absences were directly due to a physical absence from the sessions, not through participant withdrawal.

Due to a high absence rate (arguably due to the nature of clinical diagnoses), partial analyses were undertaken to examine the effects of the intervention. Although the results do shed light on the development and progression of ILSD, they do not necessarily encapsulate the variation within and between groups. Other limitations to the current study are identified as a small sample size (which was resultant of a difficulty in recruitment), samples drawn from different locations (e.g. rural versus urban) that was a result of a difficulty in recruitment; and, the division of the researcher's attention within group based intervention sessions. This meant that although each participant engaged in 15 min sessions, the amount of 1:1 time within these sessions varied dependent upon the needs of individual participants. If the sessions were run 1:1 rather than in group based settings, the rate of independent development may have been different.

The limitations to the study are representative of the challenges faced when conducting real-world research. 


\section{Conclusion}

The main findings of the current study appeared to support the suitability of the novel intervention materials in relation to the development of ILSD in young children with developmental disabilities, namely VI and DS. Remarkably, the findings also supported the materials' use in the development of ILSD within a TD context. The results also indicated that there were notable benefits for using the novel intervention materials to support ILSD development over a 10-week intervention period. A longer-term impact was also found which suggested that ILSD skills could be retained after 3 months after the intervention ceased.

Although there were identified limitations, the current project also offered contributions to the field. The study offered the first cross-syndrome comparison investigating the development of ILS in 3 distinct yet related populations (VI, DS and TD). Second, the project may shed light on the development of other ILS areas, for example mealtimes and toileting. Third, the novel intervention materials and accompanying observation schedules are the first of its kind in supporting ILSD development.

In conclusion, the paper presented a novel and innovative approach to understanding ILSD. This was done through the design and development of novel intervention materials and the employment of habilitation techniques to support ILSD. The current study is also the first to offer a contribution to dressing strategies since the early 1950s, and also the first to comparatively explore the development of ILSD in 3 distinct, yet related, participant groups (VI, DS and TD). As the current study is the first of its kind, there is a great potential toward future directions of such research in other areas of ILS, and also in relation to other clinical groups where VI can be a component or comorbidity, such as Williams Syndrome and Autism. 
Disclosure Statement: No potential conflict of interest is reported by the authors.

\section{References}

Barrett, M. D., and Bridson, A. 1983. “The effect of instructions upon children's drawings." British Journal of Developmental Psychology 1: 175-178. doi: 10.1111/j.2044-835X.1983.tb00555.x

Bishop. V. E. 2004. Teaching Visually Impaired Children. $3^{\text {rd }}$ ed. Springfield, Illinois: Charles C Thomas Publisher.

Bowman, R., Bowman, R., and Dutton, G. 2001. Disorders of vision in children: a guide for teachers and carers. London: RNIB.

http://www.ssc.education.ed.ac.uk/resources/vi\&multi/bowmandutton/bowmand utton4.html

Creavin, A. L., and Brown, R. D. 2009. "Ophthalmic Abnormalities in Children with Down Syndrome." Ophthalmology \& Strabismus 46: 76-82. doi: 10.3928/01913913-20090301-06.

Department for Education and Department of Heath England. 2015. Special educational needs and disability code of practice: 0 to 25 years. https://www.gov.uk/government/uploads/system/uploads/attachment_data/file/3 98815/SEND_Code_of_Practice_January_2015.pdf

Fairnham, M., Johnston, C., Kain, S., Kaine, N., McCauley, A., and Steele, E. 2002. "Do it yourself: Encouraging Independence in Children who are blind". In Vision Australia (blindness and low vision services), edited by Rosemary Flavel, Helen Lunn and Christine Johnston. University of Sydney.

Hayton, J. 2012. “'Get Your Coat': Developing the Independent Living Skill of Dressing in Visually Impaired Children.” MSc diss., Institute of Education, London

Hayton, J. 2015. "Developing novel intervention materials to aid dressing skills in children with Visual Impairment.” Paper presented at the Mary Kitzinger Trust Workshop, Institute of Child Health, London, November 9.

Hayton, J. 2016. “Get Your Coat: Developing Independent Dressing Skills in Young Children with Visual Impairment, Young Children with Down Syndrome and Typically Developing Children.” Paper presented at the Post Graduate Research Seminar, UCL-Institute of Education, London, November.

Herbert, M. 2003. Typical and atypical development: from conception to 
adolescence. Oxford: BPS Blackwell.

Kellegrew, D.H. 2000. “Constructing Daily Routines: A Qualitative Examination of Mothers With Young Children With Disabilities.” The American Journal of Occupational Therapy, 54: 252-259. doi: 10.5014/ajot.54.3.252

Klein, M. D. 1983. Pre-Dressing Skills: Skill Starters for Self-Help Development. Arizona: Communication Skill Builders.

Lewis, V. 1987. Development and Handicap. Oxford: Basil Blackwell.

Marsick, V.J., and Watkins, K. 2001. "Informal and Incidental Learning. ” New Directions for Adult and Continuing Education, 9: 25-34. doi: 10.1002/ace.5

Miller, O., Wall, K., and Garner, M. 2011. Quality Standards: Delivery of Habilitation Training (Mobility and Independent Living Skills) for Children and Young People with Visual Impairment. http://www.ssc.education.ed.ac.uk/resources/vi\&multi/habilitation.pdf

Moyles, J. 2015. The Excellence of Play. Berkshire: Open University Press.

Strauss, E., Sherman, E.M.S., and Spreen, O. 2006. A Compendium of Neuropsychological Tests: Administration, Norms and Commentary. Oxford University Press: New York.

Sugden, D., and Wade, M. 2013. Typical and Atypical Motor Development. Mac Keith Press

Van Herwegen, J., Rundblad, G., Davelaar, E. J., and Annaz, D. 2011. "Variability and Standardized test profiles in typically developing children with Williams Syndrome." The British Journal of Developmental Psychology, 29: 883-894. doi: 10.1111/j.2044-835X.2010.02015.X

Warren, D. 1984. Blindness and Early Childhood Development $2^{\text {nd }}$ Edition. New York: American Foundation for the Blind.

Warren, D. 1994. Blindness and Children: An Individual Differences Approach. Cambridge: Cambridge University Press. 
Table 1. Resources used in the intervention procedure

\begin{tabular}{|c|c|c|}
\hline Name & Type & Purpose \\
\hline \multirow[t]{3}{*}{ IPG } & Large jigsaw puzzle & 1. to aid familiarisation with the \\
\hline & & fastenings and practice \\
\hline & & manipulation to fasten/unfasten \\
\hline \multirow[t]{4}{*}{ Just Joey } & Interactive story using rhyming & 1. Introduce children to rhyming \\
\hline & strategies for fastening & strategies to support fastening \\
\hline & & 2. Dress along with Joey and \\
\hline & & fasten coats together \\
\hline \multirow[t]{4}{*}{ Standardised Outdoor Coat } & A scarf like item containing the & 1. to aid children in the transition \\
\hline & fastenings of an outdoor coat & between the abstract IPG to their \\
\hline & (zips, buttons and popper & actual outdoor coats \\
\hline & fastenings) & \\
\hline \multirow[t]{2}{*}{ Joey (Learn to Dress with Alex) } & Commercially & 1. Bright and colourful soft toy to \\
\hline & educational soft toy & aid the development of ILSD \\
\hline \multirow[t]{6}{*}{ Simon Says } & Popular verbal game & 1. Interactive game used as a \\
\hline & & warm-up for the children \\
\hline & & 2. Labelling body parts (i.e. put \\
\hline & & your finger on your nose) is \\
\hline & & beneficial for body awareness \\
\hline & & and postural control) \\
\hline \multirow[t]{4}{*}{ Observation Schedule } & Four continuum based schedules & 1. Scoring based on dependence \\
\hline & based on observed stages of & independence \\
\hline & fastening each item (zips, & demonstrate progression for each \\
\hline & buttons, poppers and laces). & fastening \\
\hline
\end{tabular}




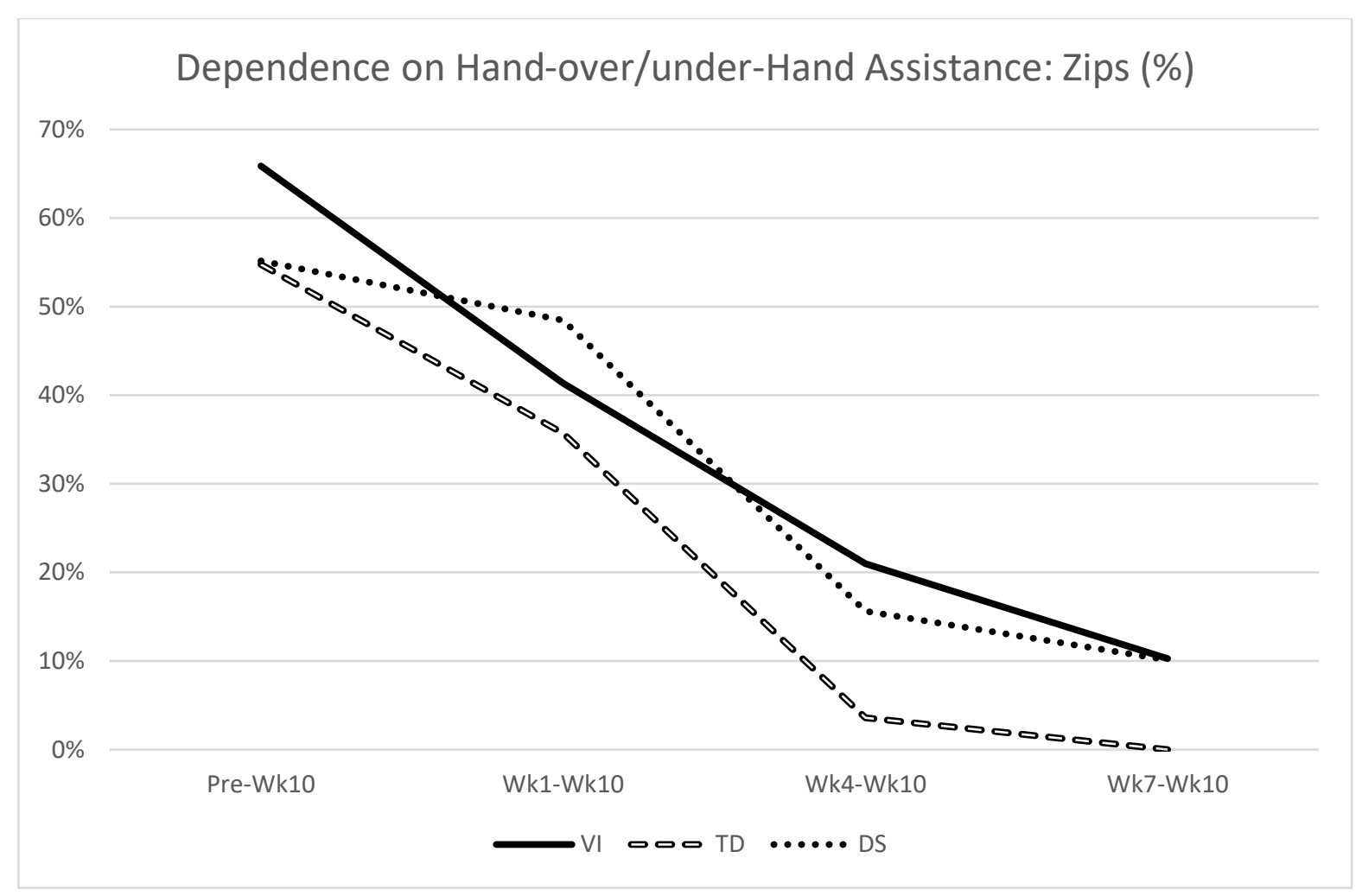

Figure 1.

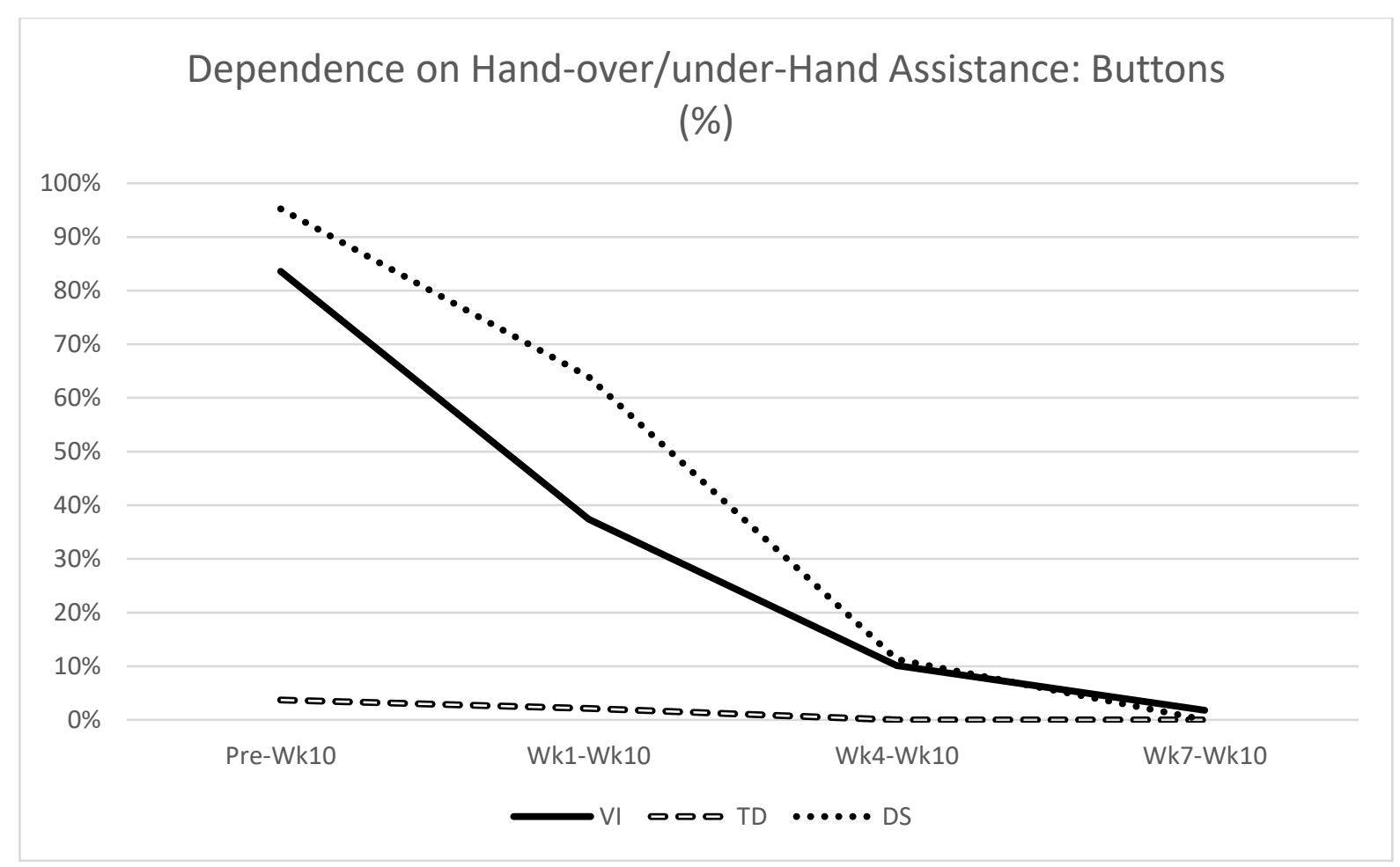

Figure 2. 
Dependence on Hand-over/under-Hand Assistance: Poppers

(\%)

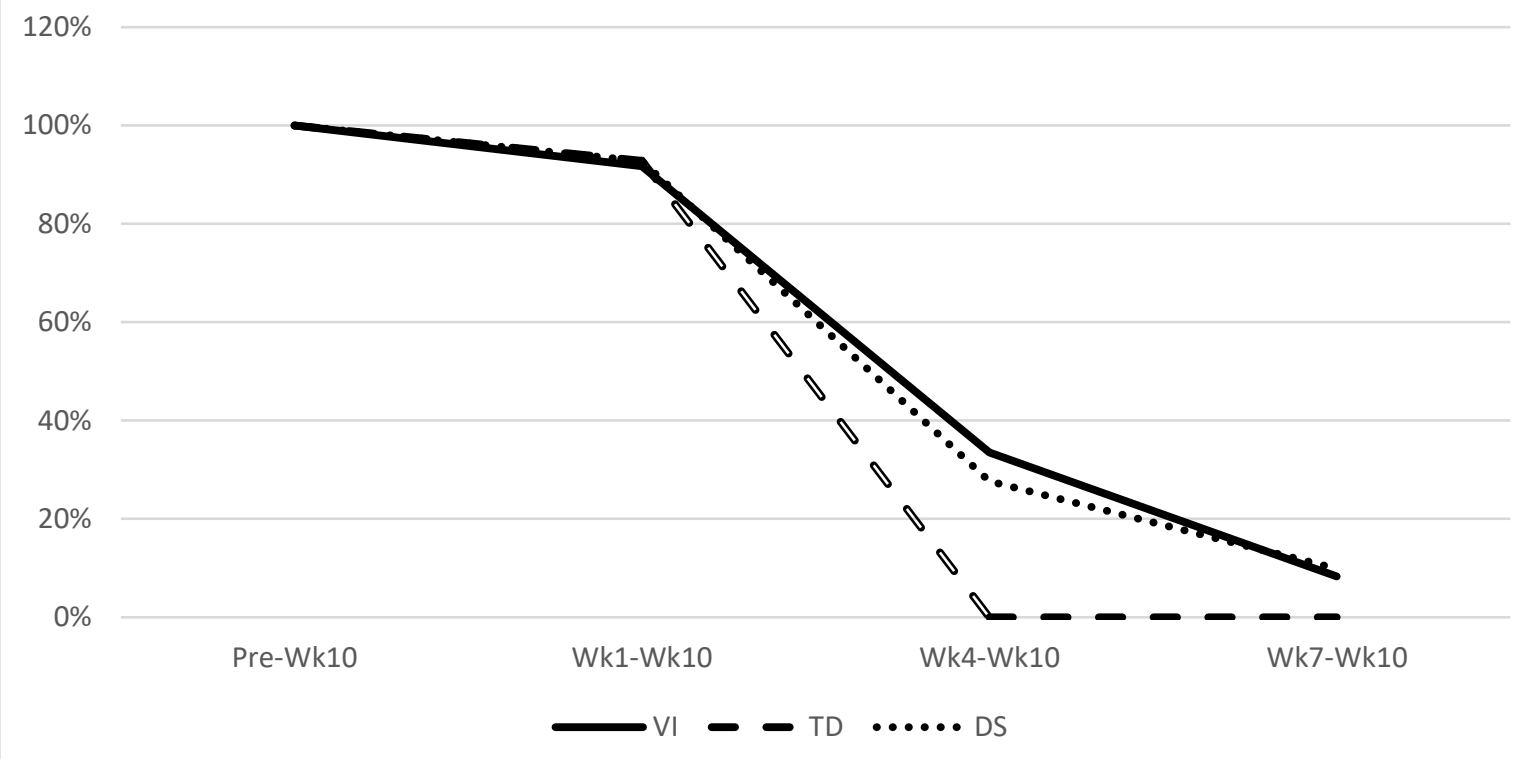

Figure 3.

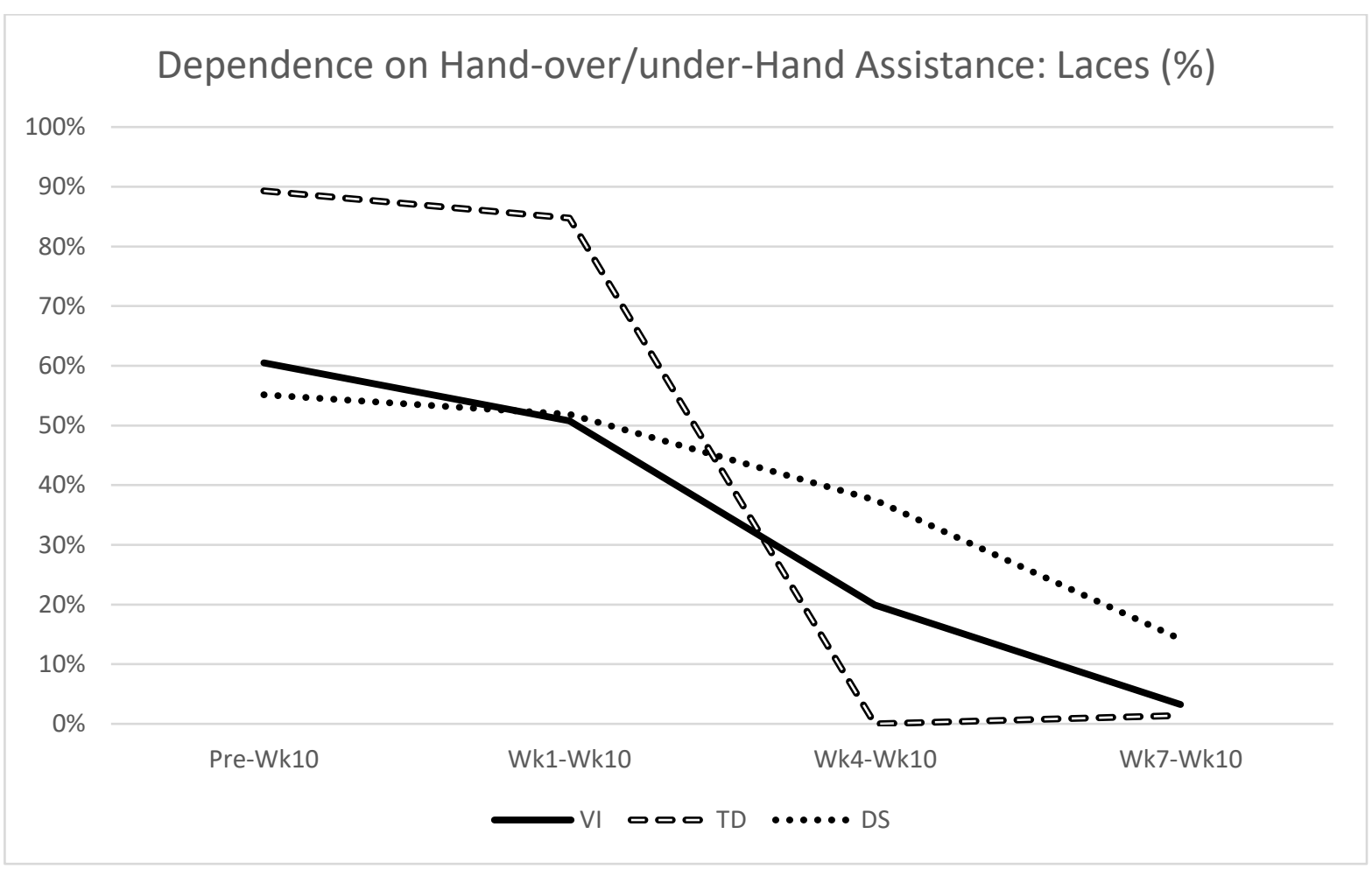

Figure 4. 
Longer-term Impact on Zip Fastening Ability (\%)

$0 \%$

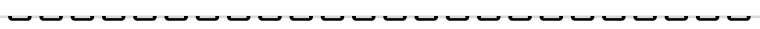

$-1 \%$

$-1 \%$

$-2 \%$

$-2 \%$

$-3 \%$

$-3 \%$

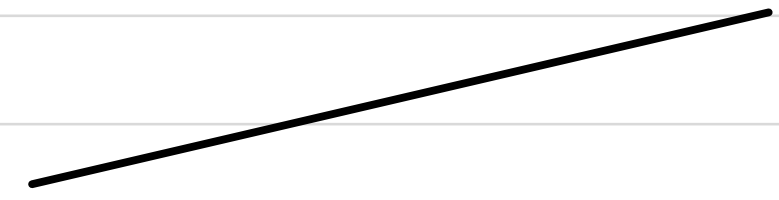

Wk10-1mnth

Wk10-3mnth

$\longrightarrow$ VI Doo TD

Figure 5.

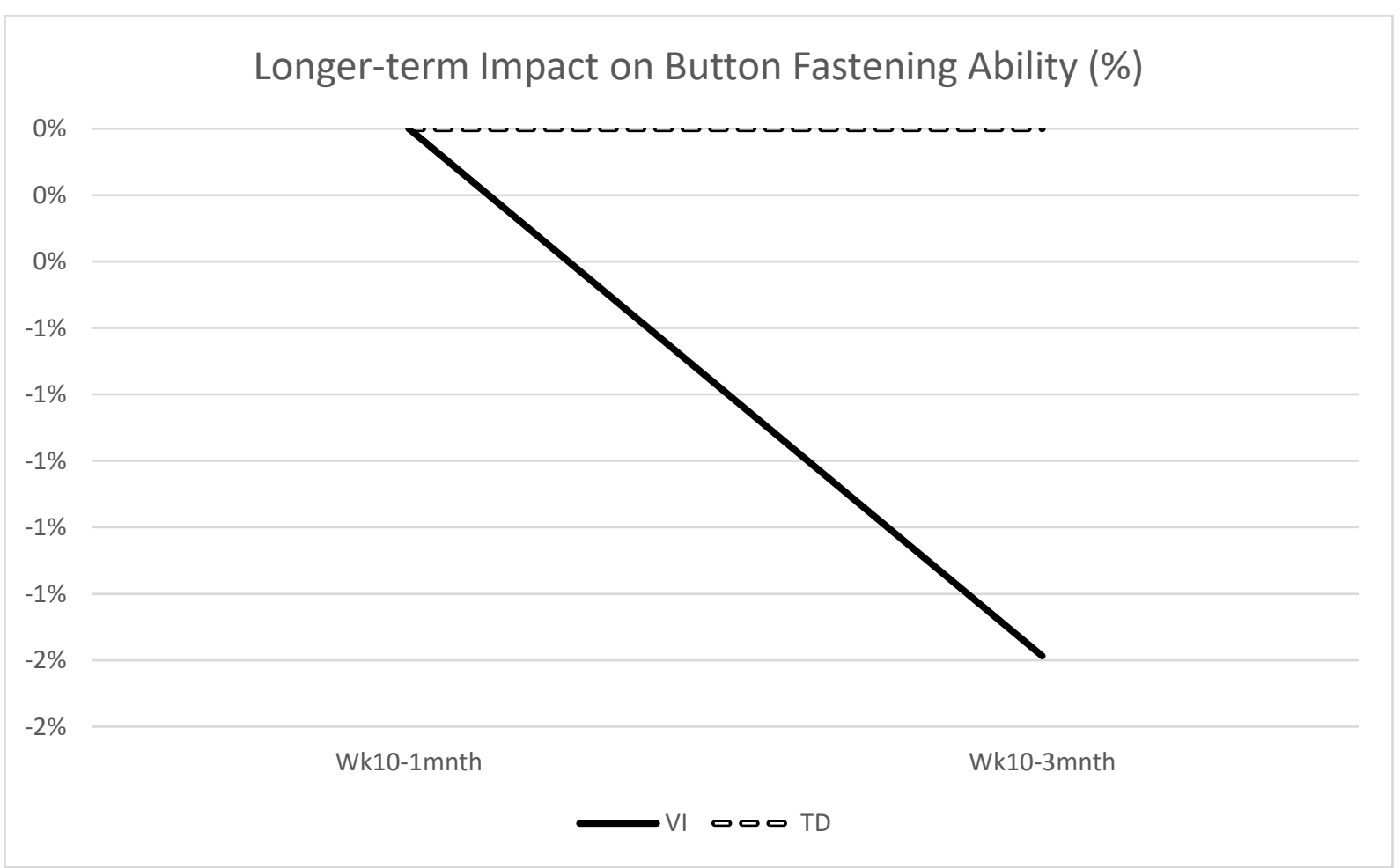

Figure 6. 


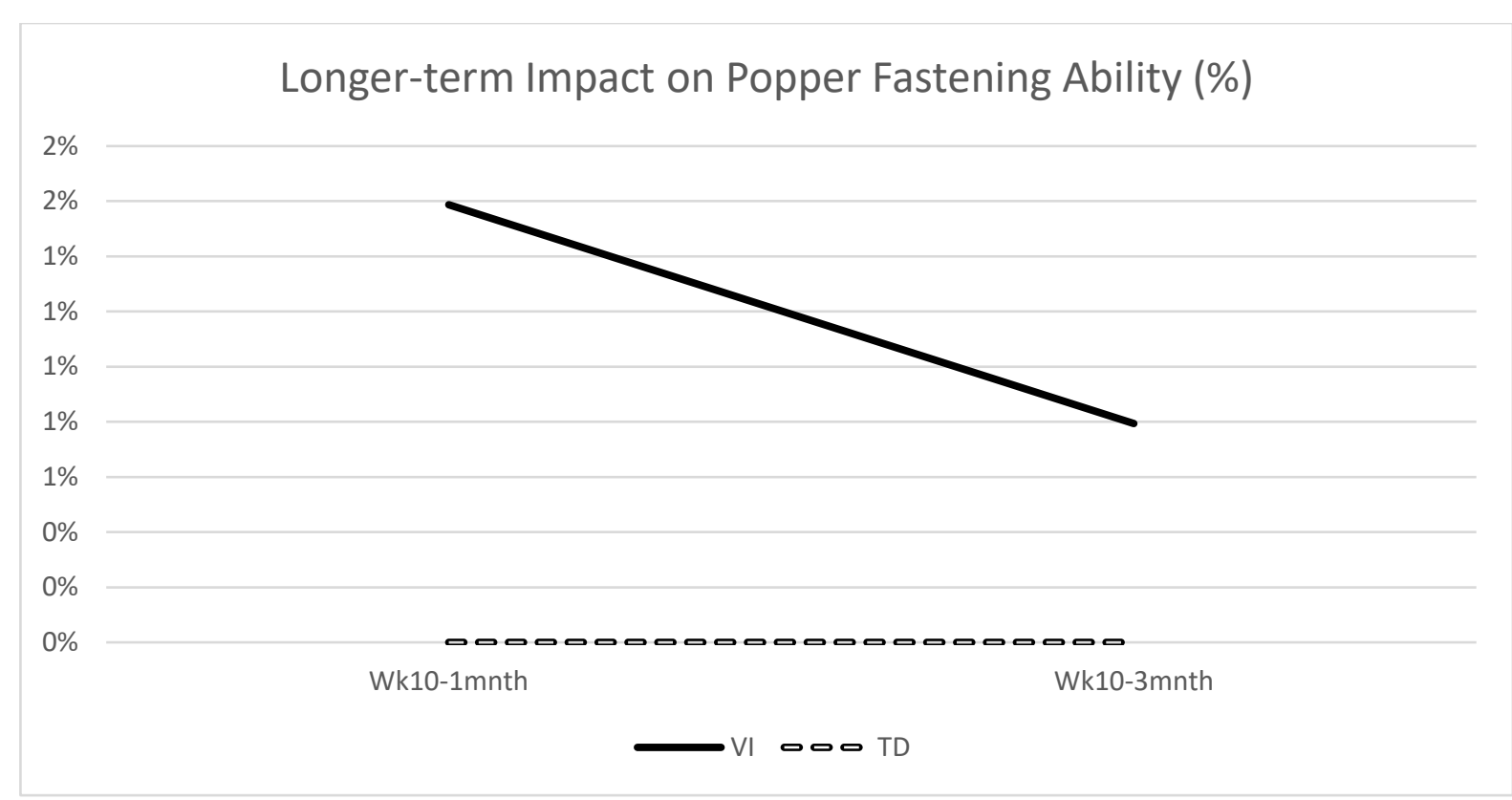

Figure 7.

Longer-term Impact on Lace Fastening Ability (\%)

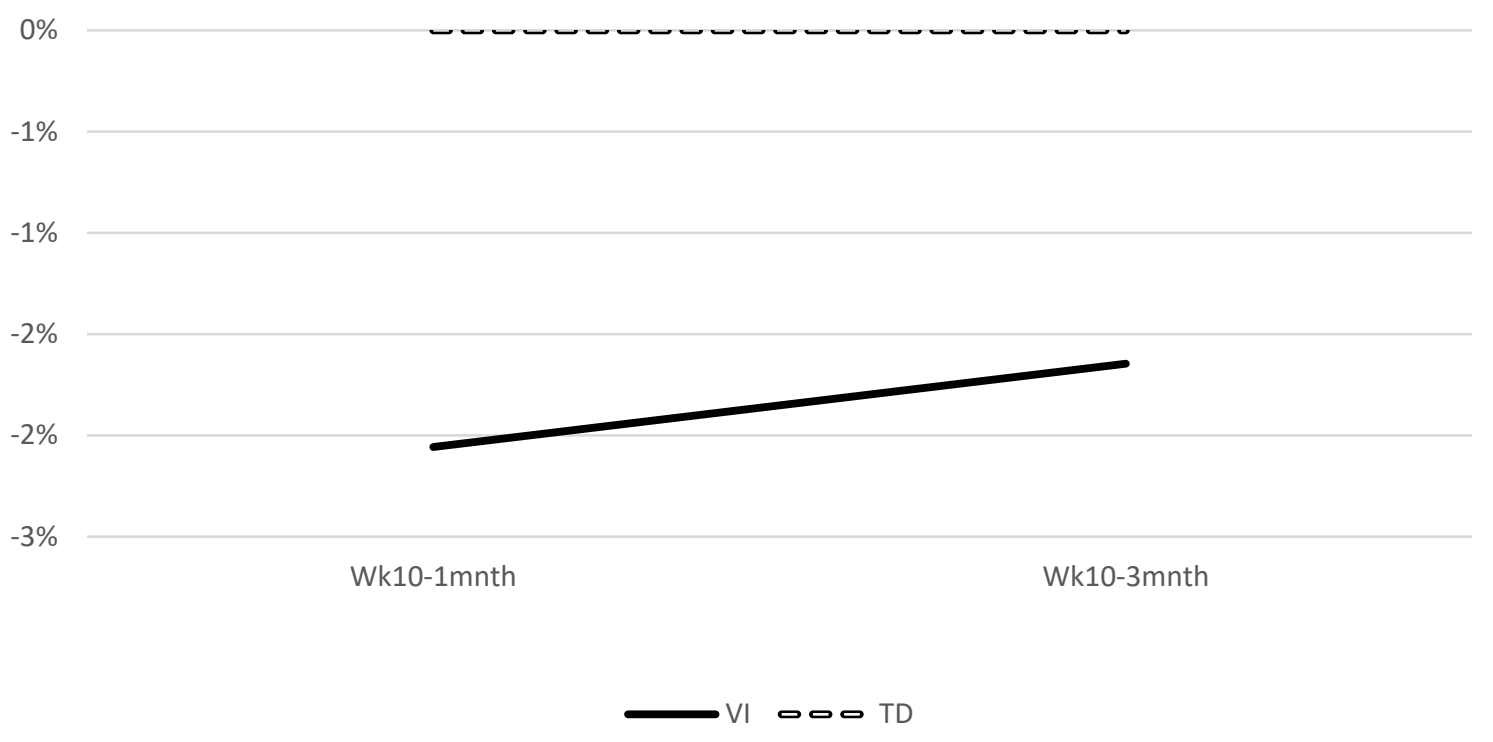

Figure 8. 
Figure Captions:

Figure 1 . The mean reduction in zip dependence scores over the 10 week intervention period for each participant group

Figure 2. The mean reduction in button dependence scores over the 10 week intervention period for each participant group

Figure 3. The mean reduction in popper dependence scores over the 10 week intervention period for each participant group

Figure 4. The mean reduction in lace dependence scores over the 10 week intervention period for each participant group

Figure 5. The impact of the intervention on zip fastening ability 1 and 3 months postintervention for VI and TD groups

Figure 6 . The impact of the intervention on button fastening ability 1 and 3 months postintervention for VI and TD groups

Figure 7. The impact of the intervention on popper fastening ability 1 and 3 months postintervention for VI and TD groups

Figure 8 . The impact of the intervention on lace fastening ability 1 and 3 months postintervention for VI and TD groups 